\section{Phacoemulsification in true exfoliation of the lens capsule: a case series}

AR Kulkarni' , J Al-Ibrahim², S Haider², S Elsherbiny³ and $\mathrm{R} \mathrm{Scott}{ }^{1}$

Keywords: true exfoliation; capsule;

phacoemulsification; cataract

Purpose We report five cases (seven eyes) of true exfoliation during an 18-month period. Of the two bilateral cases, the first was identified immediately before cataract surgery and the second spontaneously developed a split in the anterior capsule just before capsulorrhexis, mimicking a partial capsulorrhexis. In the three unilateral cases, true exfoliation was noted during the first examination. Methods Vision blue aided uneventful capsulorrhexis differentiating its edge from the true exfoliation edge, and in the first two cases, the anterior capsule was sent for histopathology and ultrasound of the fellow eye was requested.

Results Ultrasound and histopathology demonstrated lamellar separation of the anterior portion of the lens capsule, confirming the diagnosis of true exfoliation. Cataract surgery by phacoemulsification was uneventful in all cases

Conclusion True exfoliation of the lens capsule can masquerade as a partial capsulorrhexis and should be looked for before surgery and immediately before capsulorrhexis to avoid creating a partial thickness capsulorrhexis and its related surgical complications. No zonule weakness was appreciated in our cases. To our knowledge, the spontaneous occurrence of a curvilinear lamellar capsular dehiscence with a flap before capsulorrhexis has not been reported before. This series highlights that cataracts associated with true exfoliation of the lens capsule can be safely operated, with the help of vision blue, by routine phacoemulsification without having to convert to the extracapsular technique.

Eye (2007) 21, 835-837; doi:10.1038/sj.eye.6702441; published online 2 June 2006

\section{Introduction}

True exfoliation (TE) of the lens capsule is rare and easily overlooked with characteristic delamination and curling of the superficial layers into the anterior chamber. ${ }^{1,2}$ Uneventful phacoemulsification was previously reported in two cases of TE., We report a series of phacoemulsification in five cases (seven eyes) of TE. A large anterior lamellar flap was identified immediately before cataract surgery in the first case and before capsulorrhexis mimicking a partial capsulorrhexis in the second case (with no previous report found on Medline search).

Although looked for, TE was not seen previously but may have been detected by ultrasound. In the other three cases, TE was noted during their first examination. Successful phacoemulsification was carried out in all seven eyes without having to convert to the extracapsular technique.

\section{Case reports}

Case 1

A 96-year-old ex-crystal factory worker developed bilateral moderate sclerotic cataracts with no TE clinically. Her best corrected visual acuity (BCVA) was $6 / 18$ and 6/12 in the right and left eyes, respectively. A month later, immediately before cataract surgery to the right eye, a large dehiscence in the anterior capsule with an attached lamellar flap was noted waving in the anterior chamber (Figure 1a and b). A peripheral split was also noted in the left capsule (Figure 1c). Ultrasound B-scan confirmed TE with splits in the superficial layers of the left thickened capsule.
${ }^{1}$ University Hospital Birmingham NHS Trust, Selly Oak Hospital, Birmingham, UK

${ }^{2}$ The Guest Hospital, Dudley Group of Hospitals NHS Trust, West Midlands, UK

${ }^{3}$ Sandwell and West Birmingham Hospitals NHS Trust, West Midlands, UK

Correspondence: AR Kulkarni, University Hospital Birmingham, Selly Oak hospital, Raddlebarn Road, Birmingham, West Midlands B29 6JP, UK

Tel: + 441216278535 ;

Fax: +441216278865.

E-mail: ruparch2@hotmail.com

Received: 31 October 2005 Accepted in revised form: 21 April 2006 Published online: 2 June 2006 

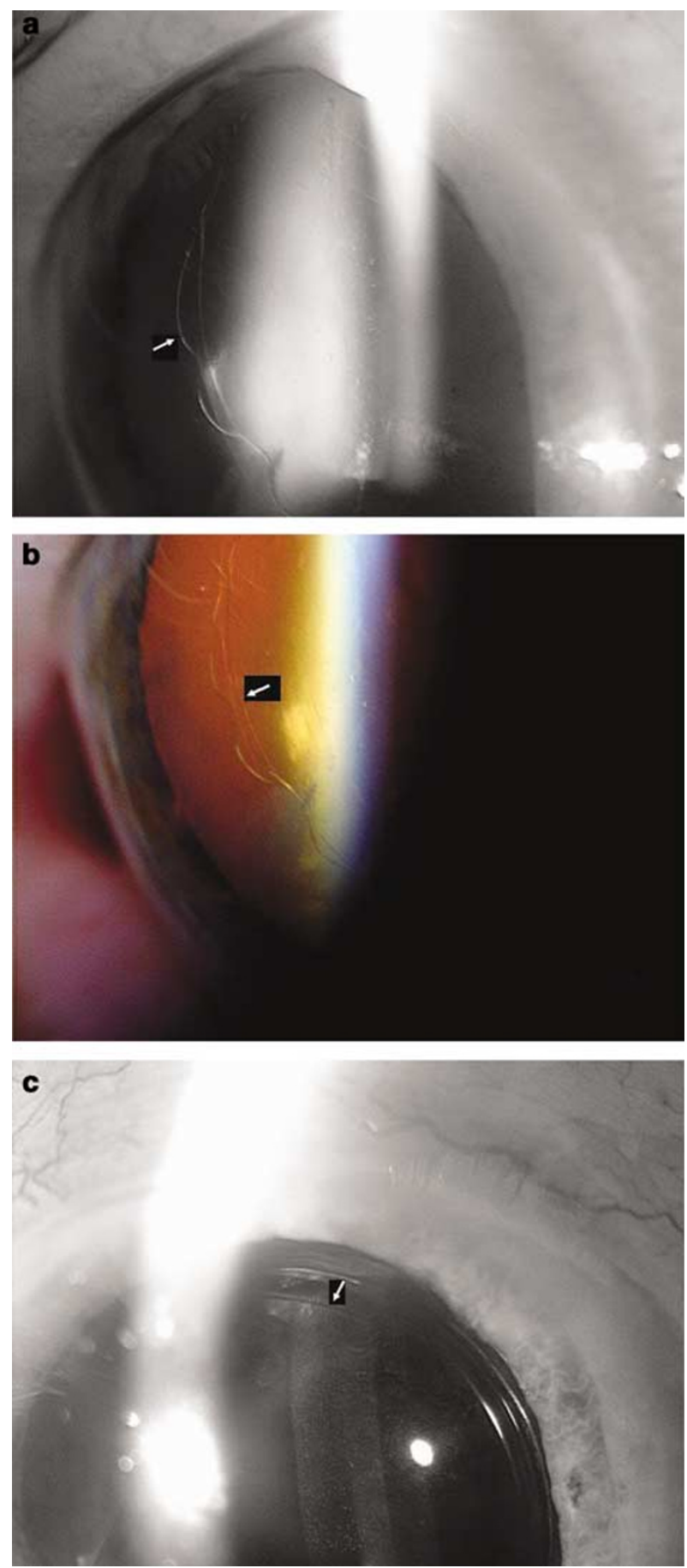

Figure 1 (a) Right eye of Case 1: Curvilinear split in the anterior lens capsule with the attached superficial flap appearing as a thin wavy membrane in the anterior chamber. (b) Right eye of Case 1: Retroillumination shows the two edges of the curvilinear dehiscence in the anterior lens capsule clearly with the superficial flap attached to the more central edge of the lamellar split. (c) Left eye of Case 1: A peripheral curvilinear lamellar dehiscence in the anterior lens capsule is seen.
The capsular dehiscence extended while injecting healon. Vision blue aided uneventful capsulorrhexis differentiating it from the TE edge. During left eye surgery, the capsulorrhexis was performed peripheral to the dehiscence making completion easier.

Histopathology of the capsule confirmed TE with PASpositive thickened lens capsule and peeling away of the outer portion of the capsule and underlying irregular degenerated monolayer epithelium.

\section{Case 2}

A 94-year-old ex-glass-factory-worker developed bilateral moderate sclerotic cataracts. Her BCVA was $6 / 12$ in either eye. Preoperatively the anterior capsule appeared normal on slit-lamp examination. Before capsulorrhexis, a crescent-shaped split with a capsular flap developed with injection of healon into the right anterior chamber. Vision blue aided capsulorrhexis, which was performed outside the dehiscence margins. Phacoemulsification was uneventful. Histopathology of the anterior capsule confirmed TE, with stretches of thickened PAS-positive lens capsule with partial peeling away of the outer portion of the lens capsule and underlying irregular, degenerated subcapsular monolayer epithelium. Although clinically not visible, TE was confirmed by ultrasound in the left eye.

\section{Case 3}

An 81-year-old ex-metal press worker had bilateral sclerotic cataracts. Her BCVA were hand movements and $6 / 12$ in the right and left eyes, respectively. A circumferential split (TE) in the right anterior capsule was noted. Capsulorrhexis was placed outside the extent of the TE margin with the help of vision blue and phacoemulsification was uneventful. The vision improved to $6 / 60$, due to dry age related macular degeneration.

\section{Case 4}

A 70-year-old ex-foundry worker with bilateral posterior subcapsular cataracts, interestingly, had TE in his left eye. After routine phacoemulsification and uneventful postoperative recovery, the BCVA improved from $6 / 18$ preoperatively to $6 / 5$ postoperatively.

\section{Case 5}

A 68-year-old ex-furnace worker was referred with bilateral nuclear sclerotic cataracts and BCVA of $6 / 18$ and 6/9 in his right and left eyes, respectively. A small $2 \mathrm{~mm}$ diameter true exfoliation of the right anterior lens 
capsule was noted. Uneventful capsulorrhexis and right phacoemulsification was performed with good visual outcome of $6 / 6$.

\section{Comment}

Since Elschnig in 1922 described TE of the lens capsule, ${ }^{5}$ resulting from heat-activated proteolysis, ${ }^{1,6,7}$ there have been at least 35 more TE cases ${ }^{1-3,5-8}$ of which only two had phacoemulsification., ${ }^{3,4}$ The 'double-ring sign' during capsulorrhexis (considered a precursor of TE), ${ }^{9}$ zonule weakness, pseudoexfoliation disease, ${ }^{10,11}$ and glaucoma ${ }^{8}$ were absent in our series.

All our patients had a history of prolonged heat exposure. In the first two cases, light microscopy confirmed a split in the thickened PAS-positive anterior capsule, similar to the findings by Majima ${ }^{4}$ and Karp. ${ }^{6}$ Brodrick $^{12}$ reported electron microscopy findings of irregular degenerated subcapsular epithelium with round nuclei and accumulation of granular aggregates and lacunae in the cytoplasm unlike those reported by Majima ${ }^{4}$ and Burde. ${ }^{8}$ Ultrasound confirmed TE preoperatively in the fellow-eye of the first two cases showing splits in the thickened anterior lens capsule centrally, with the superficial and deeper lamellae remaining adherent peripherally.

TE can masquerade as partial capsulorrhexis and should be identified to avoid creating a partial thickness capsulorrhexis and its related complications. To our knowledge, this series emphasizes the safety of phacoemulsification in cases with TE, which should be considered a routine procedure with no necessity for extracapsular surgery.

\section{Acknowledgements}

We thank Professor Luthert and Mr Robin Howes at The Department of Pathology, The Institute of Ophthalmology, London, for the histology report of true exfoliation of the lens capsule and the Department of Visual Sciences, Birmingham Midland Eye Centre, Birmingham, for the ultrasound results. Mr Prasanna Pimpalnerkar, Microsoft Certified professional for his technical support.

\section{References}

1 Meades K, Versace P. True exfoliation of the lens capsule. Aust NZ J Ophthalmol 1992; 20(4): 347-348.

2 Yamamoto N, Miyagawa A. True exfoliation of the lens capsule following uveitis. Graefes Arch Clin Exp Ophthalmol 2000; 238(12): 1009-1010.

3 Rossiter J, Morris A. Trypan blue vital staining of the anterior lens capsule in the management of cataract in true exfoliation of the lens capsule. Eye 2005; 19(7): 809-810.

4 Majima K, Kousaka M, Kanbara Y. A case of true exfoliation. Ophthalmologica 1996; 210(6): 341-343.

5 Elschnig A. Detachment of the zonular lamella in glassblowers. Klin Monatsbt Augenheilkd 1922; 69: 632-732.

6 Karp CL, Fazio JR, Culbertson WW, Green WR. True exfoliation of the lens capsule. Arch Ophthalmol 1999; 117(8): 1078-1080.

7 Anderson IL, van Bockxmeer FM. True exfoliation of the lens capsule. A clinicopathological report. Aust NZ J Ophthalmol 1985; 13(4): 343-347.

8 Cashwell Jr LF, Holleman IL, Weaver RG, van Rens GH. Idiopathic true exfoliation of the lens capsule. Ophthalmology 1989; 96(3): 348-351.

9 Ataka S, Kohno T, Kurita K, Wada S, Takahashi Y, Shiraki K. Histopathological study of the anterior lens capsule with a double-ring sign. Graefes Arch Clin Exp Ophthalmol 2004; 242(3): 245-249, [E-pub 2003 November 26].

10 Dark AJ, Streeten BW, Cornwall CC. Pseudoexfoliative disease of the lens: a study in electron microscopy and histochemistry. Br J Ophthalmol 1977; 61(7): 462-472.

11 Dvorak TG. Pseudo-exfoliation of the lens capsule: relation to true exfoliation of the lens capsule as reported in the literature and role in the production of glaucoma capsulocuticulare. Am J Ophthalmol 1954; 37(1): 1-12.

12 Brodrick JD, Tate Jr GW. Capsular delamination (true exfoliation) of the lens. Report of a case. Arch Ophthalmol 1979; 97(9): 1693-1698. 